\title{
Retinal and choroidal vascular occlusion secondary to corticosteroid embolisation
}

\author{
WILLIAM S WILKINSON, ${ }^{1}$ CRAIG M MORGAN, ${ }^{1}$ ELIYA BARUH, ${ }^{2}$ \\ AND KURT A GITTER ${ }^{3}$
}

From 'Vitreoretinal Services, the WK Kellogg Eye Center at the University of Michigan Medical Center, Ann Arbor, Michigan, the ${ }^{2}$ Ichilov Hospital, Tel Aviv, Israel, and the ${ }^{3}$ Retinal Research Foundation and Touro Infirmary, New Orleans, Louisiana, USA

SUMMARY We report a case of visual loss following intranasal injection of triamcinolone acetonide and phenylephrine hydrochloride in a 22-year-old female with chronic sinusitis. Clinical examination and fluorescein angiography revealed vascular occlusions which involved multiple small branches of both the choroidal and retinal circulations. The mechanism of such a complication probably involves inadvertent intra-arterial injection into the anterior or the posterior ethmoidal artery, with retrograde flow into the opthalmic arterial system. Precautions can be taken to avoid such complications.

Ocular complications may follow therapeutic injections around the eyes, nose, lips, and scalp. ${ }^{1-4}$ The first reports of blindness following intranasal injections of warm paraffin were decribed in the early 1900 s.' The beneficial effects of corticosteroids became apparent in the early 1960s, and instances of unilateral or bilateral visual loss immediately following extraorbital corticosteroid injections have been described subsequently. ${ }^{5}$ We report a case of transient visual loss with combined choriocapillaris and retinal arteriolar occlusion following an intranasal injection of corticosteroid and phenylephrine hydrochloride.

\section{Case report}

A 22-year-old-female with a history of chronic sinusitis was given an injection of triamcinolone acetonide (Kenalog) and phenylephrine hydrochloride in both inferior nasal turbinates. $1 \mathrm{ml}$ containing $20 \mathrm{mg}$ of trimacinolone acetonide was injected first uneventfully in the left turbinate. After waiting two minutes an additional $1 \mathrm{ml}$ with $20 \mathrm{mg}$ of triamcinolone acetonide was injected in the right turbinate. The physician encountered an unusual amount of resistance during the injection, and the injection required much more force than usual to

Correspondence to Craig $M$ Morgan, MD, Huntington Eye Associates, 1151 Hall Greer Boulevard, Huntington, West Virginia 25701, USA. complete. Immediately after the injection the patient noted a marked loss of vision in her right eye. She was seen within several hours by her ophthalmologist. Vision at that time was noted to be counting fingers in the right eye, and a $3+$ relative afferent pupillary defect was present. On ophthalmoscopy multiple foci

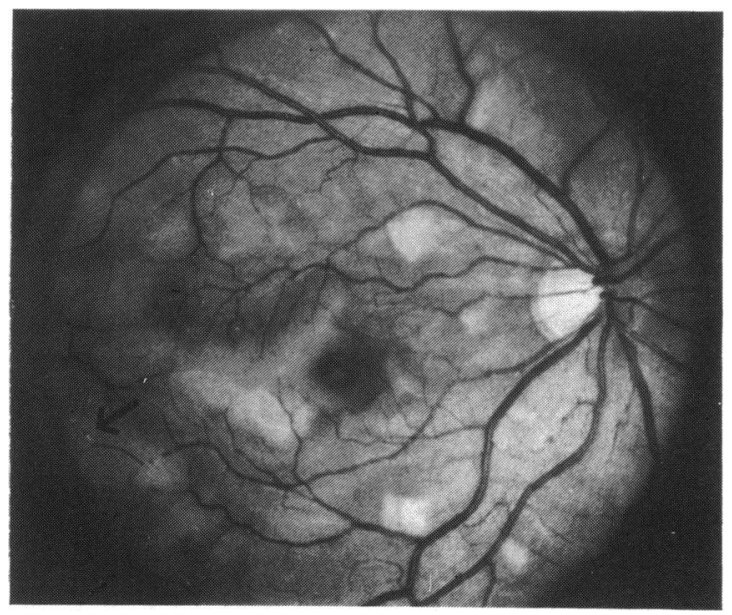

Fig. 1 Fundus photograph, right eye. Note the multiple areas of retinal ischaemia and the intra-arterial material (arrow). 


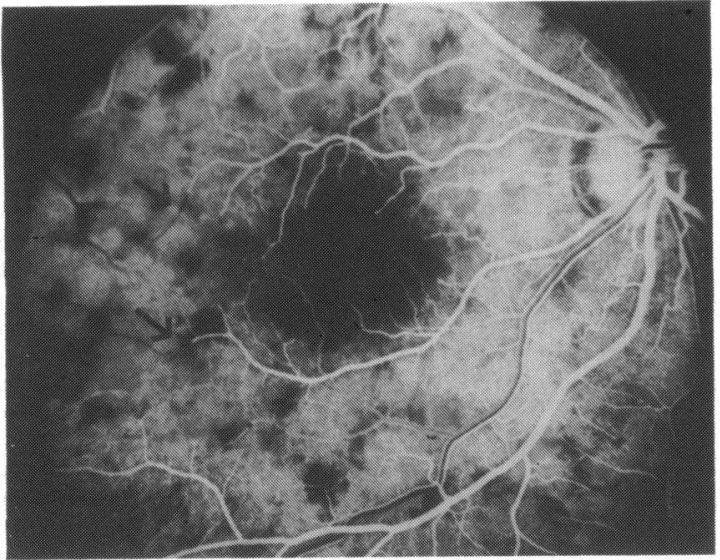

Fig. 2 Fluorescein angiogram, early arteriovenous phase. Areas of choroidal non-perfusion and retinal arteriolar obstruction (arrows) are present.

of intra-arteriolar, small, plaque-like, whitish material were seen in the temporal macular vessels of the right eye.

Twenty-four hours later she was seen by one of us (KAG). At that time the visual acuity was $20 / 400$ in the right eye and $20 / 20$ in the left. A $2+$ relative afferent pupillary defect was present in the right eye. Ophthalmoscopic examination revealed multiple areas of retinal ischaemia in the macula and the same intra-arteriolar material (Fig. 1). The retinal arterioles temporal to the macula appeared to be occluded, but the optic nerve was normal. The left eye was normal. Goldmann visual field examination revealed an inferior altitudinal defect with peripheral constriction of the remaining superior field. Fluorescein angiography (Fig. 2) in the early phases revealed several randomly scattered, small, irregular zones of choroidal hypofluorescence. In addition retinal arteriolar filling defects were present in the temporal paramacular area. Fluorescein leaked from these retinal vessels during the late phases of the fluorescein angiogram, and small hyperfluorescent zones in the choroid were present which slowly increased in size (Fig. 3). These findings were thought to be consistent with multiple microemboli to the chroroidal and retinal circulations, presumably from the local corticosteroid injection. No specific treatment was given.

Four days after the first visit the fluorescein angiogram was repeated and was found to be normal. A subsequent fluorescein angiogram two months later was within normal limits as well, and no retinal pigment epithelial changes were detected. One year later the patient was asymptomatic, had recovered $20 / 20$ vision, and had a normal visual field.

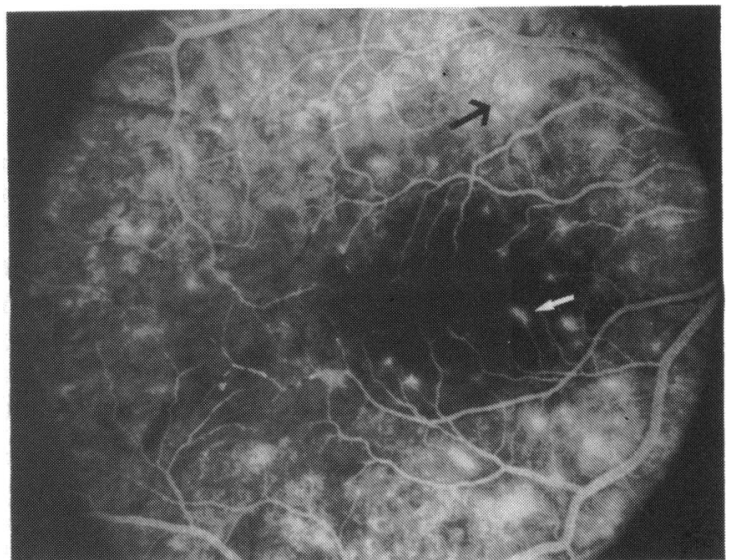

Fig. 3 Fluorescein angiogram, late phase. There is staining of some of the retinal vessels (lower arrow) and focal areas of choroidal hyperfluorescence (upper arrow).

\section{Discussion}

It is thought that retinal and choroidal emboli following injections in areas remote from the eye, such as the nose, may occur as a result of inadvertent intraarterial injection of the drug. ${ }^{14}$ The ophthalmic artery branches peripherally into the anterior and posterior ethmoidal arteries, which supply the nasal mucosa. These vessels anastomose with branches of the external carotid artery in the face. Inadvertent injection of small particles under pressure into an anterior ethmoidal artery can cause retrograde flow into the ophthalmic artery, which then can enter the eye via the central retinal artery and the short posterior ciliary arteries. ${ }^{4}$ Therapeutic agents injected under pressure into a chronically inflamed tissue with a sinusoidal vascular system may gain access to the supplying arteries as well. ${ }^{6}$

The patient described herein demonstrated nonperfusion of some of the retinal arterioles and lobules of the choriocapillaris. Corticosteroid emboli from the injection were the probable cause of these combined retinal and choroidal vascular occlusions, though vasospasm induced by the phenyephrine hydrochloride which was added to the injection solution may have been a contributing factor.

The outcome in this instance was fortunately favourable, with full visual recovery. Other workers, however, have reported unilateral and bilateral visual loss following embolisation of material injected in the head and neck. ${ }^{3}$ To avoid potential complications precautions should be taken in administering such injections. The use of a decongestant such as epinephrine or cocaine applied to the turbinates prior to the injection may be useful. ${ }^{4}$ 
Proprioceptive awareness of syringe resistance may facilitate safe injection. Aspiration prior to injection should always be performed; however, local collapse of the microvasculature may give a falsely negative aspiration, and insufficient resistance on injection may signify an intra-arterial injection. ${ }^{5}$ Injecting slowly, in small quantities, and moving the needle while injecting to avoid placing a large bolus of material in one location have been advocated. ${ }^{4}$ In addition, when possible, using suspensions that have small particles may be beneficial, as suspensions with larger particles are more likely to cause significant vascular occlusion should embolisation occur. ${ }^{45}$

The authors acknowledge the help of Bonnie Turner in the preparation of this manuscript.

\section{References}

1 Van Bahr G. Multiple embolisms in the fundus of an eye after injection in the scalp. Acta Ophthalmol (Kbh) 1963; 41: 85-91.

2 Ellis PP. Visual loss following tonsilectomy: possible association with injections in tonsillar fossae. Arch Ophthalmol 1968; 87: 12830.

3 Wilson RS, Havener WH, McGrew RN. Bilateral retinal artery and choriocapillaris occlusion following injection of long-acting corticosteroid suspensions in combination with other drugs: I. Clinical studies. Ophthalmology 1978; 85: 967-73.

4 Byers B. Blindness secondary to steroid injections into the nasal turbinates. Arch Ophthalmol 1979; 97: 79-80.

5 Thomas EL, Laborde RP. Retinal and choroidal vascular occlusion following intralesional corticosteroid injection of a chalazion. Ophthalmology 1986; 93: 405-7.

6 Whiteman DW, Rosen DA, Pinkerton RMH. Retinal and choroidal microvascular embolism after intranasal corticosteroid injection. Am J Ophthalmol 1980; 89: 851-3.

Accepted for publication 5 November 1987. 